Research Article

\title{
Galloping Control for Iced Conductors Using Tuned Mass Dampers with Fixed Time-Delayed Feedback
}

\author{
Guo Li $(\mathbb{D}$, Li Li $\mathbb{D}$, and Peng Zhu $\mathbb{D}$ \\ School of Civil Engineering \& Mechanics, Huazhong University of Science \& Technology, Wuhan 430074, China \\ Correspondence should be addressed to Li Li; 13476294116@163.com
}

Received 4 September 2018; Revised 6 January 2019; Accepted 30 January 2019; Published 27 February 2019

Academic Editor: Gabriele Cazzulani

Copyright (@ 2019 Guo Li et al. This is an open access article distributed under the Creative Commons Attribution License, which permits unrestricted use, distribution, and reproduction in any medium, provided the original work is properly cited.

\begin{abstract}
A tuned mass damper with fixed time-delay feedbacks (TTMD) is first applied to suppress galloping of iced conductors. It is different from general time-delay feedback control, which also connects an added mass to a galloping body and can absorb vibrational energy. The two-degrees-of-freedom model captures the essential mechanical behaviors of iced conductors with TTMD undergoing galloping. The harmonic balance method is then employed to obtain the approximate analytical solutions of the system. Particularly, the expressions of galloping amplitude and angle frequency were obtained. Then the influence regulations of the time-delay parameter $T$ and strength parameter $K$ on galloping control are fully analysed. The results show the added fixed time-delay feedback control can promote the TTMD's effect, when suitable time-delay parameter $T$ and strength parameter $K$ are chosen. Moreover, the response amplitude and natural frequency change periodically with time-delay parameter $T$. With strength parameter $K$ increasing, the response amplitude increases or decreases for a fixed value of $T$ and the TTMD's effect can be enhanced.
\end{abstract}

\section{Introduction}

Galloping of iced conductors is a kind of self-excited vibration, due to aerodynamic instability. It appears with low frequency $(0.1 \sim 3 \mathrm{~Hz})$ and large amplitude $(5 \sim 300$ times the diameter of wire). The large displacements induced by galloping can produce great energy and cause serious electric accidents, such as, flashover, line breaking, and tower collapse which are serious accidents of power grids. Since galloping was observed in the 1930s, extensive researches have been carried on this issue, and many passive and active devices have been equipped to suppress galloping, for example, spacer, detuning pendulum, spoiler, and tuned mass damper (TMD) [1-6].

Among them, the TMD has received particular attention, which prevents galloping by increasing damping energy dissipation of system. Although the TMDs have been widely used in vibration control of building, bridge and mechanical systems, and acquired expected vibration control effect in some applications, their efficiency is not high enough in galloping control of iced conductors. Previous studies are mainly devoted to design the spring-viscous connection of the added mass to the system for reaching a suitable tuning to the frequency of an assigned mode [7-11]. TMD devices may need to be improved.

Moreover, the time-delayed feedback also plays an important role in vibration control [12]. And the timedelayed feedback control has been adopted to control the vibration of lathe cutting tools and container cranes $[13,14]$. Motivated by them, one comes up with an idea that tuned mass dampers with time-delayed feedback control (TTMD) may be another way to increase the energy dissipating of the system. Many researches have been developed around timedelayed feedback control. Mehmood et al. [15] studied the oscillating control of an elastically mounted rigid cylinder undergoing vortex-induced vibrations by linear and nonlinear active velocity feedback controllers. Wang et al. [16] explored the effect of time-delayed feedback on galloping response of square prisms and found that the time-delayed feedback force plays an important role in controlling galloping responses. Dai et al. [17] investigated the effectiveness of linear and nonlinear time-delayed feedback controls to 
galloping amplitude of an elastically mounted square cylinder. Ravichandran et al. [18] studied the nonlinear resonance in Duffing oscillators with fixed and integrative timedelayed feedbacks. The effects of delayed feedback control on a nonlinear vibration absorber system have been investigated by Zhao and $\mathrm{Xu}$ [19]. However, the system of iced conductors with TTMD is different from them. The timedelayed feedback controller is set between galloping body and added mass. It has the function of a time-delayed feedback controller and an absorber.

This paper thus designs a TMD device with a fixed time-delayed feedback control and studies its effect in galloping control of iced conductors [20]. The remaining parts are organized as follows: three simplified models approximating the galloping of different dynamical systems are presented in the Mathematical Model section. In the Asymptotic Solution section, the first-order harmonic balance method is used to obtain the approximate analytical solutions of the proposed models. The Numerical Results and Discussion section gives numerical results, that is, the variation of galloping amplitude for different systems with respect to time-delay parameter $T$ and strength parameter $K$. Finally, the results are discussed and summarized.

\section{Mathematical Model}

2.1. Simplified Models. Following the papers [9, 15, 21], galloping of iced conductors-TTMD system can be simplified to the model shown in Figure 1(a): a cylinder body $\left(m_{1}\right)$ connected with a small added mass $\left(m_{2}\right)$ is mounted by an elastic spring $\left(k_{1}\right)$ and a dashpot $\left(c_{1}\right)$, which is subjected to a steady flow. The added mass is connected to the galloping body by an elastic spring $\left(k_{2}\right)$ and a viscous damper $\left(c_{2}\right)$. The system is completed with a time-delayed controller $(K)$ which is situated between the galloping body and the added mass. Moreover, the aerodynamic force acting on the added mass is assumed to be negligible in comparison with that acting on the galloping body.

Similarly, galloping of iced conductors-TMD system can be simplified to the model shown in Figure 1(b): a galloping body immersed in an incoming flow is supported by an elastic spring and a dashpot. In addition, an added mass is connected to the galloping body by an elastic spring, which is supposed to be barely affected by the aforementioned flow. A viscous damper is also set between the cylinder body and the added mass. And the galloping of iced conductors system can be simplified to the model shown in Figure 1(c): a cylinder body is immersed in a steady flow, which is mounted by an elastic spring and a dashpot.

2.2. Governing Equations. The vertical motion of the iced conductors-TTMD system can be described by the following delayed differential equations:

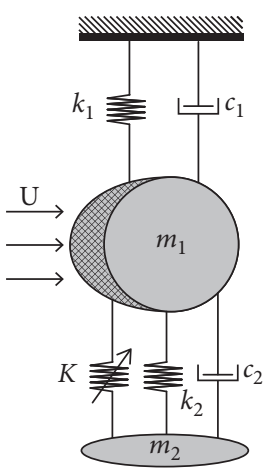

(a)

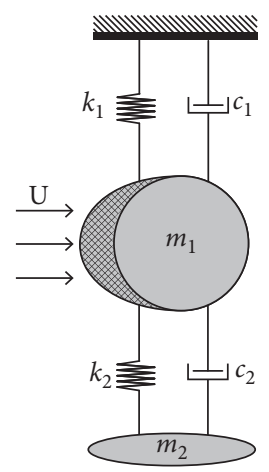

(b)

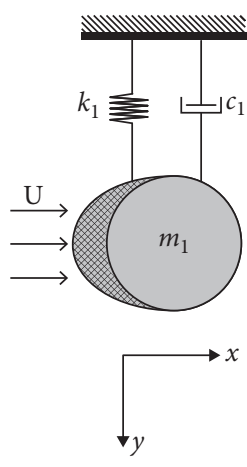

(c)
FIgURE 1: Simplified model of iced conductors (a) with TTMD system, (b) with TMD system, and (c) without added absorber.

$$
\begin{aligned}
& m_{1} \ddot{y}_{1}+c_{1} \dot{y}_{1}+k_{1} y_{1}+c_{2}\left(\dot{y}_{1}-\dot{y}_{2}\right)+k_{2}\left(y_{1}-y_{2}\right) \\
& \quad+K\left(y_{1}-y_{2}(t-T)\right)=f_{y}, \\
& m_{2} \ddot{y}_{2}+c_{2}\left(\dot{y}_{2}-\dot{y}_{1}\right)+k_{2}\left(y_{2}-y_{1}\right)+K\left(y_{2}(t-T)-y_{1}\right)=0,
\end{aligned}
$$

where $y_{1}$ denotes the position of the galloping body; $y_{2}$ is the position of added mass, with respect to the zero equilibrium position of the system; $m_{1}$ is the equivalent mass of the iced conductors; $m_{2}$ is the equivalent mass of the added mass; $k_{1}$ is the equivalent stiffness of the iced conductors; $k_{2}$ is the stiffness of the linear spring, which connects $m_{2}$ to $m_{1} ; c_{1}$ is the equivalent damping coefficient of the iced conductors; $c_{2}$ is the damping coefficient of the viscous damper, which is set between $m_{1}$ and $m_{2} ; K$ is the strength parameter of the timedelayed feedback; $T$ is the time-delay parameter; and $f_{y}$ is the vertical wind load intensity and the dot symbol stands for differentiation with respect to time $t$.

As for the aerodynamic force $f_{y}$, some assumptions need to be introduced: (1) the quasi-steady theory is adopted and (2) the ice is uniformly distributed along the transmission line. Following Barrero-Gil et al. [21], Belvins [22], and Luongo et al. [23], a cubic polynomial is used to approximate the vertical fluid force coefficient so that the aerodynamic force can be expressed as

$$
f_{y}=\frac{1}{2} \rho U^{2} D\left(a_{1} \frac{\dot{y}_{1}}{U}+a_{3}\left(\frac{\dot{y}_{1}}{U}\right)^{3}\right),
$$

where $\rho$ is the air density, $U$ is the uniform flow velocity, $D$ is the diameter of the conductors, $\dot{y}_{1} / U$ is the angle of attack, and $a_{1}$ and $a_{3}$ are the aerodynamic coefficients of the drag force, which are measured via wind tunnel tests.

Similarly, the vertical motion of the iced conductorsTMD system can be described by the following differential equations:

$$
\begin{aligned}
m_{1} \ddot{y}_{1}+c_{1} \dot{y}_{1}+k_{1} y_{1}+c_{2}\left(\dot{y}_{1}-\dot{y}_{2}\right)+k_{2}\left(y_{1}-y_{2}\right) & =f_{y}, \\
m_{2} \ddot{y}_{2}+c_{2}\left(\dot{y}_{2}-\dot{y}_{1}\right)+k_{2}\left(y_{2}-y_{1}\right) & =0 .
\end{aligned}
$$


And the vertical motion of the iced conductors galloping system can be described by the following differential equation:

$$
m_{1} \ddot{y}_{1}+c_{1} \dot{y}_{1}+k_{1} y_{1}=f_{y} .
$$

In particular, the parameters in equations (3) and (4) are the same with those in equation (1).

2.3. Asymptotic Solution. The governing equations can be solved by either analytical method or numerical method. Compared to numerical solutions, the analytic solutions can present the varying tendencies of the amplitude and frequency of galloping. Since galloping is a periodic motion under steady state, the harmonic balance method (first order) is used to solve the equations aforementioned.

2.4. Galloping Response for Iced Conductors-TTMD System. Taking into consideration of equations (3) and (4) and introducing a set of dimensionless variables $q_{1}=y_{1} / D, q_{2}=$ $y_{2} / D, \quad \omega_{1}=\sqrt{k_{1} / m_{1}}, \quad \gamma=\sqrt{k_{2} / k_{1}}, \quad \chi=\sqrt{K / k_{1}}, \quad \mu=m_{2} / m_{1}$, $\zeta_{1}=c_{1} / 2 m_{1} \omega_{1}, \zeta_{E}=c_{2} / 2 m_{1} \omega_{1}, m^{*}=m_{1} / \rho D^{2}, U^{*}=U / \omega_{1} D$, $\tau=\omega_{1} t$, and $T^{*}=\omega_{1} T$, the dimensionless governing equations for the iced conductors-TTMD system can be written as

$$
\begin{aligned}
& q_{1}^{\prime \prime}+2 \zeta_{1} q_{1}^{\prime}+q_{1}+2 \zeta_{E}\left(q_{1}^{\prime}-q_{2}^{\prime}\right)+\gamma^{2}\left(q_{1}-q_{2}\right) \\
& \quad+\chi^{2}\left(q_{1}-q_{2}\left(\tau-T^{*}\right)\right)=\frac{U^{* 2}}{2 m^{*}}\left(a_{1} \frac{q_{1}^{\prime}}{U^{*}}+a_{3}\left(\frac{q_{1}^{\prime}}{U^{*}}\right)^{3}\right), \\
& \mu q_{2}^{\prime \prime}+2 \zeta_{E}\left(q_{2}^{\prime}-q_{1}^{\prime}\right)+\gamma^{2}\left(q_{2}-q_{1}\right)+\chi^{2}\left(q_{2}\left(\tau-T^{*}\right)-q_{1}\right)=0,
\end{aligned}
$$

where $q_{1}$ and $q_{2}$ are the nondimensional vertical displacements of the galloping body and added mass, $\omega_{1}$ is the undamped frequency ratio, $\zeta_{1}$ is the dimensionless parasitic damping coefficient, $\zeta_{E}$ is the dimensionless damping coefficient of the viscous damper, $\gamma$ is the frequency ratio, $\chi$ is the dimensionless strength parameter of time-delayed feedback control, $T^{*}$ is the dimensionless time-delay parameter, $m^{*}$ is the mass ratio of the galloping body, $\mu$ is the ratio between galloping body and added mass, $U^{*}$ is the reduced velocity, $\tau$ is the dimensionless time, and the prime represents differentiation with respect to the dimensionless time.

Assuming that the steady response is harmonic, the solutions of equation (5) have the following forms:

$$
\begin{aligned}
& q_{1}=A \sin \left(\omega^{*} \tau\right), \\
& q_{2}=B \sin \left(\omega^{*} \tau+\phi\right),
\end{aligned}
$$

where the four unknowns, $\omega^{*}=\omega / \omega_{1}, A, B$, and $\phi$ need to be solved.

Substituting equation (6) into equation (5), one obtains

$$
\begin{aligned}
& -A \omega^{* 2} \sin \left(\omega^{*} \tau\right)+2 \zeta_{1} A \omega^{*} \cos \left(\omega^{*} \tau\right)+A \sin \left(\omega^{*} \tau\right) \\
& +2 \zeta_{E}\left(A \omega^{*} \cos \left(\omega^{*} \tau\right)-B \omega^{*} \cos \left(\omega^{*} \tau+\phi\right)\right) \\
& +\gamma^{2} A \sin \left(\omega^{*} \tau\right)-\gamma^{2} B \sin \left(\omega^{*} \tau+\phi\right) \\
& +\chi^{2}\left(A \sin \left(\omega^{*} \tau\right)-B \sin \left(\omega^{*}\left(\tau-T^{*}\right)+\phi\right)\right) \\
& =\frac{U^{* 2}}{2 m^{*}}\left(a_{1} \frac{A \omega^{*} \cos \left(\omega^{*} \tau\right)}{U^{*}}+a_{3}\left(\frac{A \omega^{*} \cos \left(\omega^{*} \tau\right)}{U^{*}}\right)^{3}\right), \\
& -\mu B \omega^{* 2} \sin \left(\omega^{*} \tau+\phi\right)+2 \zeta_{E}\left(B \omega^{*} \cos \left(\omega^{*} \tau+\phi\right)\right. \\
& \left.-A \omega^{*} \cos \left(\omega^{*} \tau\right)\right)+\gamma^{2}\left(B \sin \left(\omega^{*} \tau+\varphi\right)-A \sin \left(\omega^{*} \tau\right)\right) \\
& +\chi^{2}\left(B \sin \left(\omega^{*}\left(\tau-T^{*}\right)+\phi\right)-A \sin \left(\omega^{*} \tau\right)\right)=0 .
\end{aligned}
$$

Equating the cosine terms and sine terms about $\omega^{*} \tau$ to zero in equation (7), respectively, the following set of equations can be obtained

$$
\begin{aligned}
& 2\left(\zeta_{1}+\zeta_{E}-\frac{U^{*} a_{1}}{4 m^{*}}\right) A \omega^{*}-2 \zeta_{E} B \omega^{*} \cos \phi-\gamma^{2} B \sin \phi \\
& -\chi^{2} B\left(\sin \phi \cos \omega^{*} T^{*}-\cos \phi \sin \omega^{*} T^{*}\right)=\frac{3 a_{3} A^{3} \omega^{* 3}}{8 m^{*} U^{*}} \\
& \left(1+\gamma^{2}+\chi^{2}-\omega^{* 2}\right) A+2 \zeta_{E} B \omega^{*} \sin \phi-\gamma^{2} B \cos \phi \\
& \quad-\chi^{2} B\left(\cos \phi \cos \omega^{*} T^{*}+\sin \phi \sin \omega^{*} T^{*}\right)=0 \\
& -\mu B \omega^{* 2} \cos \phi-2 \zeta_{E} B \omega^{*} \sin \phi+\gamma^{2} B \cos \phi \\
& \quad+\chi^{2} B\left(\cos \phi \cos \omega^{*} T^{*}+\sin \phi \sin \omega^{*} T^{*}\right)-\gamma^{2} A \\
& \quad-\chi^{2} A=0, \\
& -\mu B \omega^{* 2} \sin \phi+2 \zeta_{E} B \omega^{*} \cos \phi+\gamma^{2} B \sin \phi \\
& +\chi^{2} B\left(\sin \phi \cos \omega^{*} T^{*}-\cos \phi \sin \omega^{*} T^{*}\right)-2 \zeta_{E} A \omega^{*}=0
\end{aligned}
$$

Combining equations (8c) and (8d) and eliminating both $\sin \phi$ and $\cos \phi$, one can obtain

$$
\begin{aligned}
\lambda & =\frac{B}{A} \\
& =\frac{\sqrt{\left(\gamma^{2}+\chi^{2}\right)^{2}+4 \zeta_{E}^{2} \omega^{* 2}}}{\sqrt{\left(\gamma^{2}-\mu \omega^{* 2}+\chi^{2} \cos \omega^{*} T^{*}\right)^{2}+\left(2 \zeta_{E} \omega^{*}-\chi^{2} \sin \omega^{*} T^{*}\right)^{2}}} .
\end{aligned}
$$

Combining equations (8c) and (8d) and eliminating either $\sin \phi$ or $\cos \phi$, the following equations can be obtained: 


$$
\begin{aligned}
\sin \phi & =\frac{2 \zeta_{E} \omega^{*}\left(\gamma^{2}-\mu \omega^{* 2}+\chi^{2} \cos \omega^{*} T^{*}\right)-\left(\gamma^{2}+\chi^{2}\right)\left(2 \zeta_{E} \omega^{*}-\chi^{2} \sin \omega^{*} T^{*}\right)}{\sqrt{\left(\gamma^{2}+\chi^{2}\right)^{2}+4 \zeta_{E}^{2} \omega^{* 2}} \sqrt{\left(\gamma^{2}-\mu \omega^{* 2}+\chi^{2} \cos \omega^{*} T^{*}\right)^{2}+\left(2 \zeta_{E} \omega^{*}-\chi^{2} \sin \omega^{*} T^{*}\right)^{2}}}, \\
\cos \phi & =\frac{\left(\gamma^{2}-\mu \omega^{* 2}+\chi^{2} \cos \omega^{*} T^{*}\right)\left(\gamma^{2}+\chi^{2}\right)+2 \zeta_{E} \omega^{*}\left(2 \zeta_{E} \omega^{*}-\chi^{2} \sin \omega^{*} T^{*}\right)}{\sqrt{\left(\gamma^{2}+\chi^{2}\right)^{2}+4 \zeta_{E}^{2} \omega^{* 2}} \sqrt{\left(\gamma^{2}-\mu \omega^{* 2}+\chi^{2} \cos \omega^{*} T^{*}\right)^{2}+\left(2 \zeta_{E} \omega^{*}-\chi^{2} \sin \omega^{*} T^{*}\right)^{2}}}
\end{aligned}
$$

From equations (8b) and (8c), one can obtain

$$
1-\omega^{* 2}-\mu \omega^{* 2} \lambda \cos \phi=0
$$

Equation (11) can be solved numerically along with equation (10), yielding $\omega^{*}$.

Combining equations (8a) and (8d), the amplitude response $A$ is

$$
A^{2}=\frac{4 U^{*}}{3 a_{3} \omega^{* 2}}\left(4 m^{*}\left(\zeta_{1}-\frac{1}{2} \mu \omega^{*} \lambda \sin \phi\right)-a_{1} U^{*}\right) .
$$

2.5. Galloping Response for Iced Conductors-TMD System. Compared to iced conductors-TTMD system, the iced conductors-TMD system is without time-delayed feedback control. Having this change, the nondimensional governing equations for the iced conductors-TMD system stand as such

$$
\begin{aligned}
& q_{1}^{\prime \prime}+2 \zeta_{1} q_{1}^{\prime}+q_{1}+2 \zeta_{E}\left(q_{1}^{\prime}-q_{2}^{\prime}\right)+\gamma^{2}\left(q_{1}-q_{2}\right) \\
&=\frac{U^{* 2}}{2 m^{*}}\left(a_{1} \frac{q_{1}^{\prime}}{U^{*}}+a_{3}\left(\frac{q_{1}^{\prime}}{U^{*}}\right)^{3}\right), \\
& \mu q_{2}^{\prime \prime}+2 \zeta_{E}\left(q_{2}^{\prime}-q_{1}^{\prime}\right)+\gamma^{2}\left(q_{2}-q_{1}\right)=0 .
\end{aligned}
$$

If the term $\chi^{2}=0$ in equation (5), equation (5) can be reduced to equation (13). Then the asymptotic solutions of equation (13) can be obtained by reducing the solutions of equation (5), which are as follows:

$$
\begin{gathered}
A^{2}=\frac{4 U^{*}}{3 a_{3} \omega^{* 2}}\left(4 m^{*}\left(\zeta_{1}-\frac{1}{2} \mu \omega^{*} \lambda \sin \phi\right)-a_{1} U^{*}\right), \\
1-\omega^{* 2}-\mu \omega^{* 2} \lambda \cos \phi=0 .
\end{gathered}
$$

Though equations (14) and (15) are different from the results Vicente-Ludlam et al. [24] obtained in form, they are essentially in good agreement with each other.

The expression of galloping amplitude for the iced conductors-TMD system is same as that of the iced conductors-TTMD system. The expressions of $\lambda$, sin $\phi$, and $\cos \phi$ just change from equations (9) and (10) to the following equations:

$$
\begin{aligned}
\lambda & =\frac{B}{A}=\frac{\sqrt{\gamma^{4}+4 \zeta_{E}^{2} \omega^{* 2}}}{\sqrt{\left(\gamma^{2}-\mu \omega^{* 2}\right)^{2}+4 \zeta_{E}^{2} \omega^{* 2}}}, \\
\sin \phi & =\frac{-2 \zeta_{E} \mu \omega^{* 3}}{\sqrt{\gamma^{4}+4 \zeta_{E}^{2} \omega^{* 2}} \sqrt{\left(\gamma^{2}-\mu \omega^{* 2}\right)^{2}+4 \zeta_{E}^{2} \omega^{* 2}}},
\end{aligned}
$$

$$
\cos \phi=\frac{\gamma^{2}\left(\gamma^{2}-\mu \omega^{* 2}\right)+4 \zeta_{E}^{2} \omega^{* 2}}{\sqrt{\gamma^{4}+4 \zeta_{E}^{2} \omega^{* 2}} \sqrt{\left(\gamma^{2}-\mu \omega^{* 2}\right)^{2}+4 \zeta_{E}^{2} \omega^{* 2}}}
$$

2.6. Galloping Response for Iced Conductors System. Compared to iced conductors-TTMD system and iced conductors-TMD system, the iced conductors galloping system only consists of the galloping body. The nondimensional governing equation for the iced conductors galloping system is written as

$$
q_{1}^{\prime \prime}+2 \zeta_{1} q_{1}^{\prime}+q_{1}=\frac{U^{* 2}}{2 m^{*}}\left(a_{1} \frac{q_{1}^{\prime}}{U^{*}}+a_{3}\left(\frac{q_{1}^{\prime}}{U^{*}}\right)^{3}\right) \text {. }
$$

Taking a similar manipulation as conducted for the iced conductors-TMD system, one can obtain

$$
A^{2}=\frac{4 U^{*}}{3 a_{3}}\left(4 m^{*} \zeta_{1}-a_{1} U^{*}\right) .
$$

The solution for iced conductors galloping system also coincides with the result Barrero-Gil et al. [21] obtained.

\section{Numerical Results and Discussion}

To illustrate the effect of TTMD in galloping control of iced conductors, the same parameters of the three models are selected as follows: the equivalent mass of iced conductors is $m_{1}=0.44 \mathrm{~kg}$; the equivalent stiffness of conductors is $k_{1}=156.18 \mathrm{~N} / \mathrm{m}$; the equivalent damping ratio of conductors is $\xi_{1}=0.0013$; the mass of the added mass is $m_{2}=0.2 m_{1}$; the stiffness of the linear spring is $k_{2}=0.1 k_{1}$, which is set between galloping body and added mass; the damping coefficient of the viscous damper is $c_{2}=0.5 c_{1}$; the strength parameter of time-delayed feedback is $K=0.16 k_{2}$; the timedelay parameter is $T=0.28 \mathrm{~s}$; the gravitational acceleration is $g=9.81 \mathrm{~m} \cdot \mathrm{s}^{-2}$; the air density is $\rho=1.29 \mathrm{~kg} \cdot \mathrm{m}^{-3}$; and the aerodynamic coefficients are $a_{1}=2.3$ and $a_{3}=-18$.

3.1. Comparison of the Three Systems. To verify the asymptotic solutions, a comparison between the numerical solutions and analytic solutions of equations (1)-(3) is conducted. Figure 2 presents the variation of galloping amplitude of iced conductors $\left(m_{1}\right)$ with respect to wind speed for different systems. Figure 3 gives the variation of vibration amplitude of added mass $\left(m_{2}\right)$ with respect to wind speed for different systems. It is followed from Figures 2 and 3 that the analytical solutions of $m_{1}$ and $m_{2}$ are in agreement with those from the numerical simulation for all the three systems. Therefore, it can be inferred that the asymptotic 


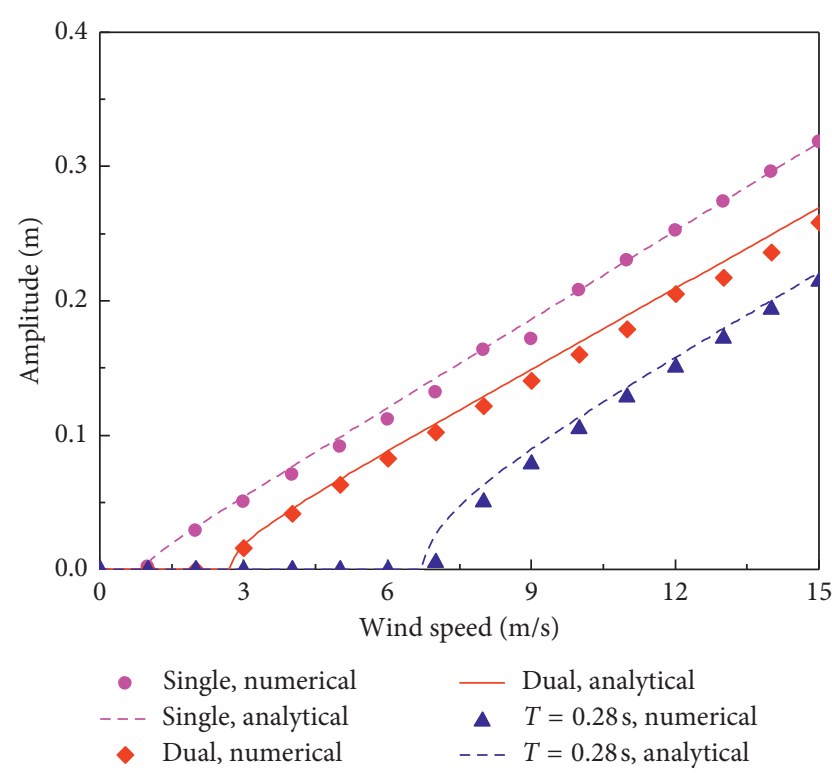

FIGURE 2: Variation of galloping amplitude of $m_{1}$ with wind speed for different systems.

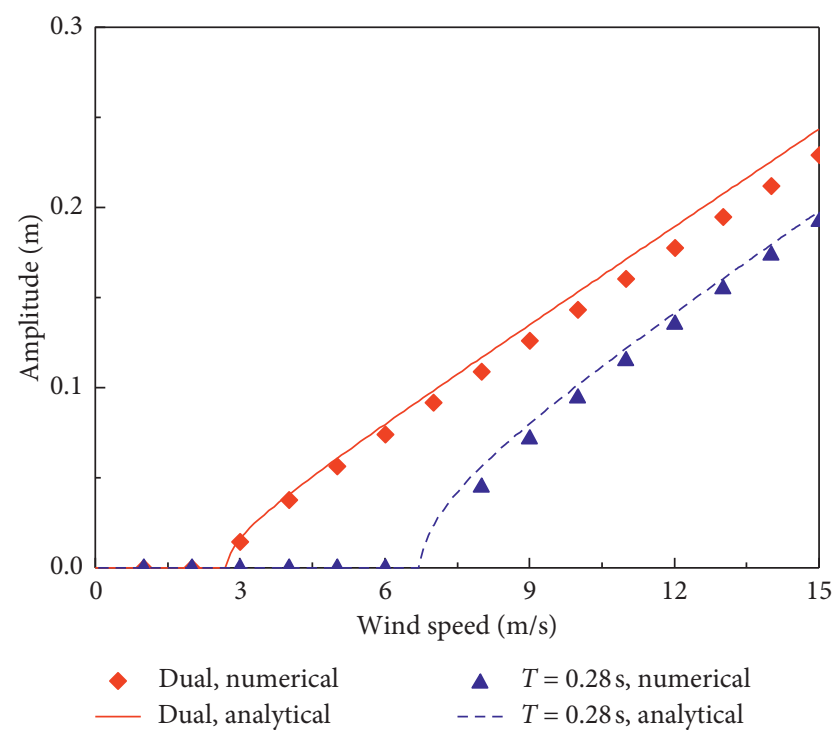

FIgURE 3: Variation of vibration amplitude of $m_{2}$ with wind speed for different systems.

solution is valid. In the subsequent analysis, the asymptotic solution proposed is adopted.

Moreover, it can be seen from Figure 2 that the galloping amplitude of $m_{1}$ for iced conductors-TMD system is lower than that of iced conductors system and the critical wind velocity of galloping for iced conductors-TMD system is higher than that of iced conductors system, which indicate that TMD can suppress galloping. One can also find from Figure 2 that the galloping amplitude of the $m_{1}$ for iced conductors-TTMD system is lower than that of iced conductors-TMD system and the critical wind velocity of galloping for iced conductors-TTMD system is higher than that of iced conductors-TMD system, which suggest the time-delayed feedback can improve the effect of TMD in galloping control. The vibration amplitude of $m_{2}$ for iced conductors-TTMD system is also lower than that of iced conductors-TMD system (Figure 3 ). In the next section, the influence of the time-delay parameter $T$ and strength parameter $K$ on galloping control is investigated.

\subsection{Effects of the Time-Delay Parameter on Galloping} Amplitude. Figure 4 gives the curves of variation of galloping amplitude with time-delay parameter $T$ under different strength parameters $K$ of time-delayed feedback. The galloping amplitude varies periodically with time-delay parameter $T$. When $0<T<0.16 \mathrm{~s}$, the response amplitude of the system with TTMD is higher than that of the system with TMD. When $0.16 \mathrm{~s}<T<0.305 \mathrm{~s}$, the response amplitude of the system with TTMD is lower than that of the system with TMD. When $0.305 \mathrm{~s}<T<0.47 \mathrm{~s}$, the behavior is the same as that of the range from 0 to $0.16 \mathrm{~s}$. It indicates that the effect of time-delay parameter $T$ on galloping control is periodic on $T$. In certain range, the galloping amplitude can be suppressed, and the time-delayed feedback can improve TMD's effect. However, in other range, the time-delayed feedback can reduce TMD's effect. In other words, the TTMD choosing suitable $T$ and $K$ may have better effect in preventing galloping than TMD. Moreover, it also can be seen from Figure 4 that the galloping amplitude of iced conductors-TTMD system changes dramatically as the strength parameter $K$ increases.

Figure 5 presents the curves of the variation of vibration amplitude of $m_{2}$ with time-delay parameter Tunder different strength parameters $K$. And the variation trends of vibration amplitude of $m_{2}$ with time-delay parameter $T$ and strength parameters $K$ are the same to those of $m_{1}$. In addition, the angle frequency of iced conductors-TTMD system varies periodically with time-delay parameter $T$ and it is larger than that of the system with TMD (Figure 6).

3.3. Effects of the Strength Parameter on Galloping Amplitude. Figure 7 presents the variation of galloping amplitude with wind speed under different time-delay parameters $T$ and strength parameters $K$. When $T=0.24 \mathrm{~s}$, the galloping amplitude of iced conductors-TTMD is lower than that of the system with TMD, and the galloping amplitude of iced conductors-TTMD system decreases with the strength parameter $K$ increasing. When $T=0.07 \mathrm{~s}$, the galloping amplitude of the system with TTMD is higher than that of the system with TMD, and the galloping amplitude of iced conductorsTTMD system increases with the strength parameter $K$ increasing. It means that the increasing of strength parameter $K$ not always decreases the galloping amplitude, but always promotes the effect of TTMD. The variation trend of vibration amplitude of $m_{2}$ with time-delay parameter $T$ under different strength parameters $K$ is the same as that of $m_{1}$ (Figure 8).

3.4. Response Amplitude vs. Tand $K$. To further understand the effect of time-delay parameter $T$ and strength parameter $K$ on response amplitude, two three-dimensional diagrams that have the variation of response amplitude with $T$ and $K$ are presented in Figures 9 and 10, respectively. It can be seen 


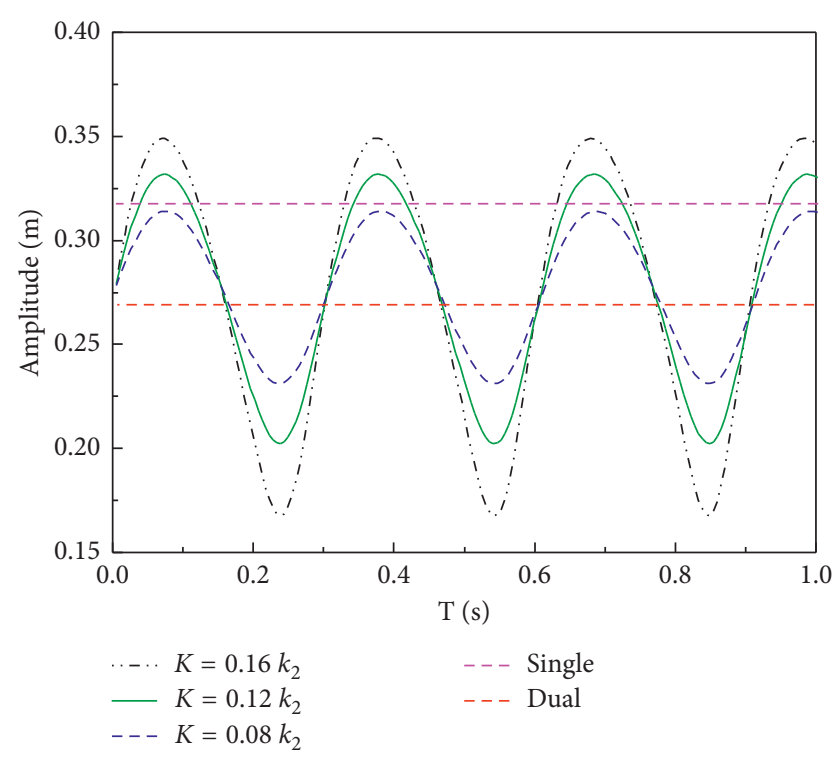

FIGURE 4: Variation of galloping amplitude of $m_{1}$ with time-delay parameter $T$ under different strength parameter $K$.

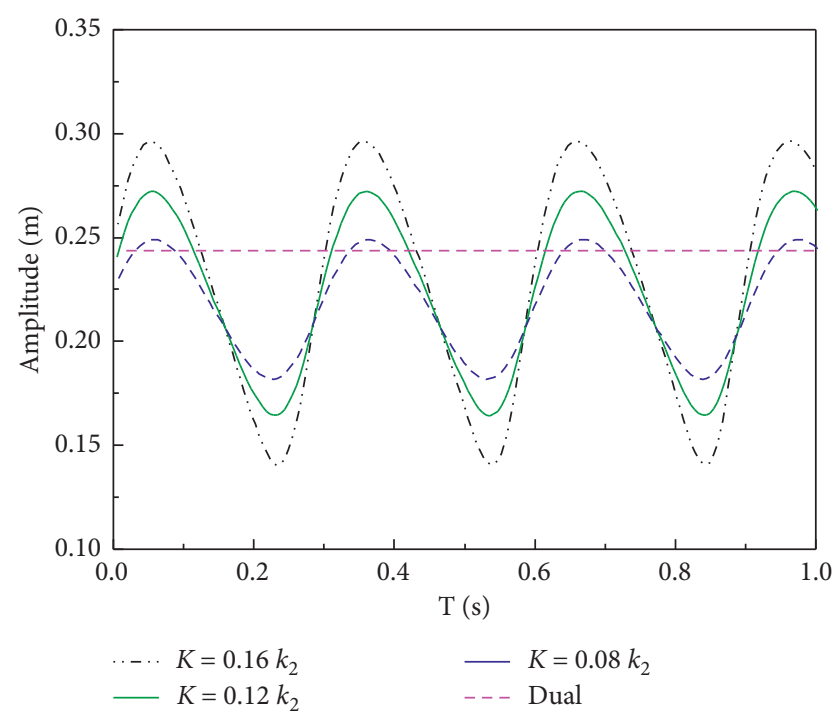

FIGURE 5: Variation of vibration amplitude of $m_{2}$ with time-delay parameter $T$ under different strength parameters $K$.

from Figure 9 that the amplitude of galloping body $\left(m_{1}\right)$ changes periodically with parameter $T$ for a fixed value of $K$. For a range of values of $T$, the amplitude of $m_{1}$ increases with $K$ increasing. For others, the amplitude of $m_{1}$ decreases with $K$ increasing. However, the strength parameter $K$ is a relatively small numerical value in practical engineering and cannot increase infinitely. The variation trend of vibration amplitude of the added mass $\left(m_{2}\right)$ with time-delay parameter $T$ and strength parameter $K$ is the same as that of $m_{1}$ (Figure 10).

3.5. Effect of Geometrical Nonlinearity on Accuracy of Response Amplitude. The galloping of iced conductors is a nonlinearity problem, and it mainly includes geometric

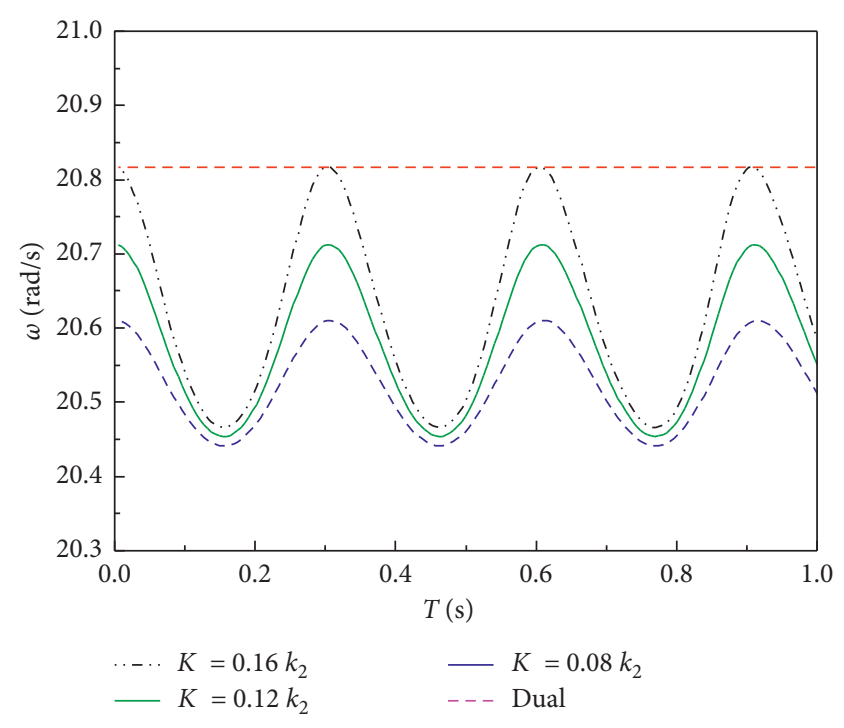

FIgURE 6: Variation of angle frequency with time-delay parameter $T$ under different strength parameters $K$.

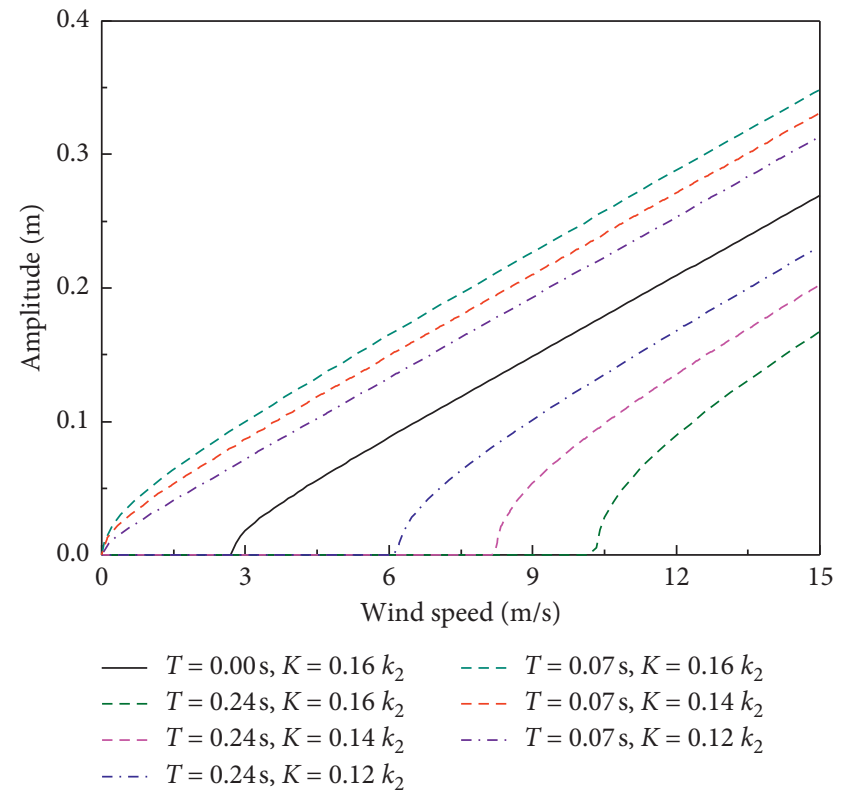

FIGURE 7: Variation of galloping amplitude with wind speed under different time-delay parameters $T$ and strength parameters $K$.

nonlinearity and aerodynamic nonlinearity [4, 25]. However, the linear equations which are used above only consider aerodynamic nonlinearity. So the comparison between the result considering geometric nonlinearity (nonlinear solution) and the solution without considering geometric nonlinearity (linear solution) is developed in this section. The project example is selected, which was used by Zhang et al. [26]. The span length of the conductors is $l=125.9 \mathrm{~m}$, the initial static horizontal tension is $H=30000 \mathrm{~N}$, the mass of unit-length iced conductors is $m=2.379 \mathrm{~kg} \cdot \mathrm{m}^{-1}$, the diameter of the conductors is $D=0.0286 \mathrm{~m}$, the area of cross section is $A=423.24 \times 10^{-6} \mathrm{~m}^{2}$, the modulus is $E=4.78033 \times 10^{10} \mathrm{~Pa}$, the damping ratio is $\xi=0.515 \times 10^{-3}$, 


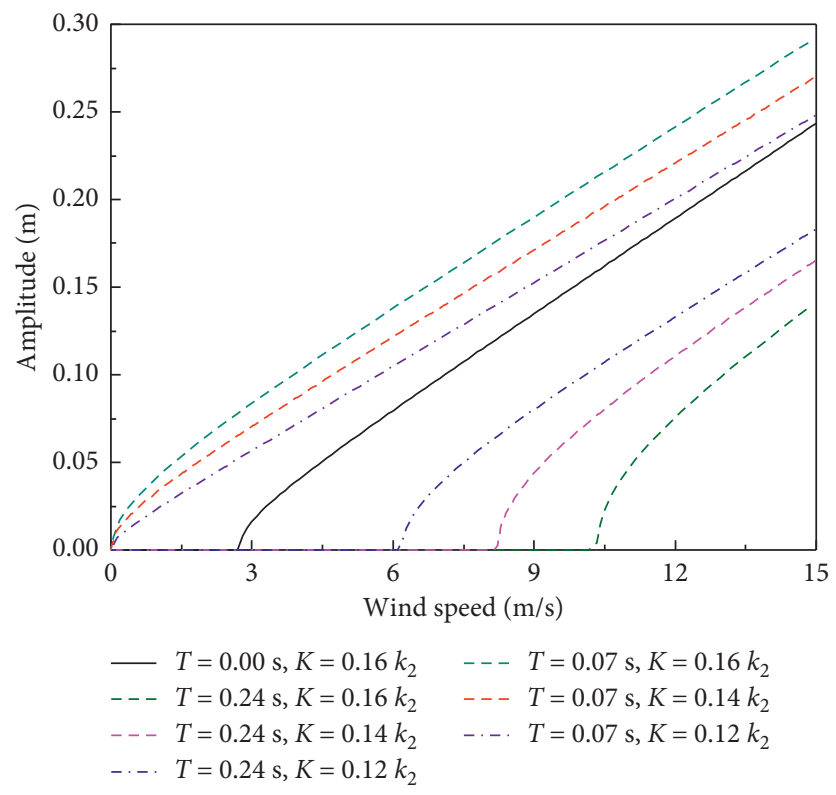

FIgURE 8: Variation of vibration amplitude of $m_{2}$ with wind speed under different time-delay parameters $T$ and strength parameters $K$.

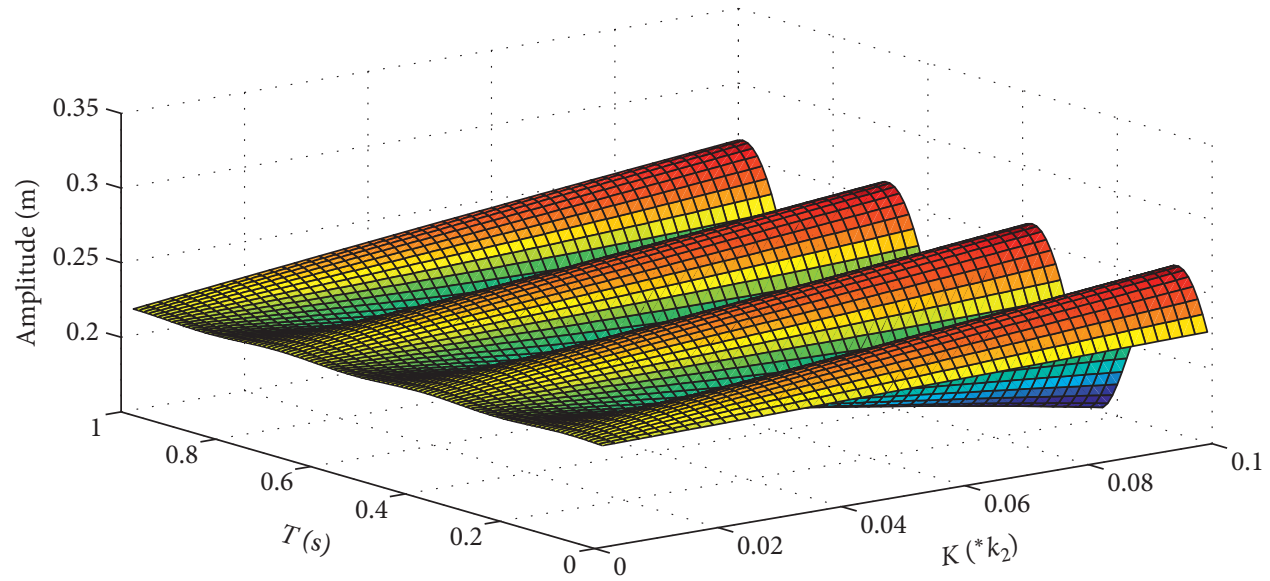

FIgURE 9: Variation of galloping amplitude of $m_{1}$ with time-delay parameter $T$ and strength parameter $K$.

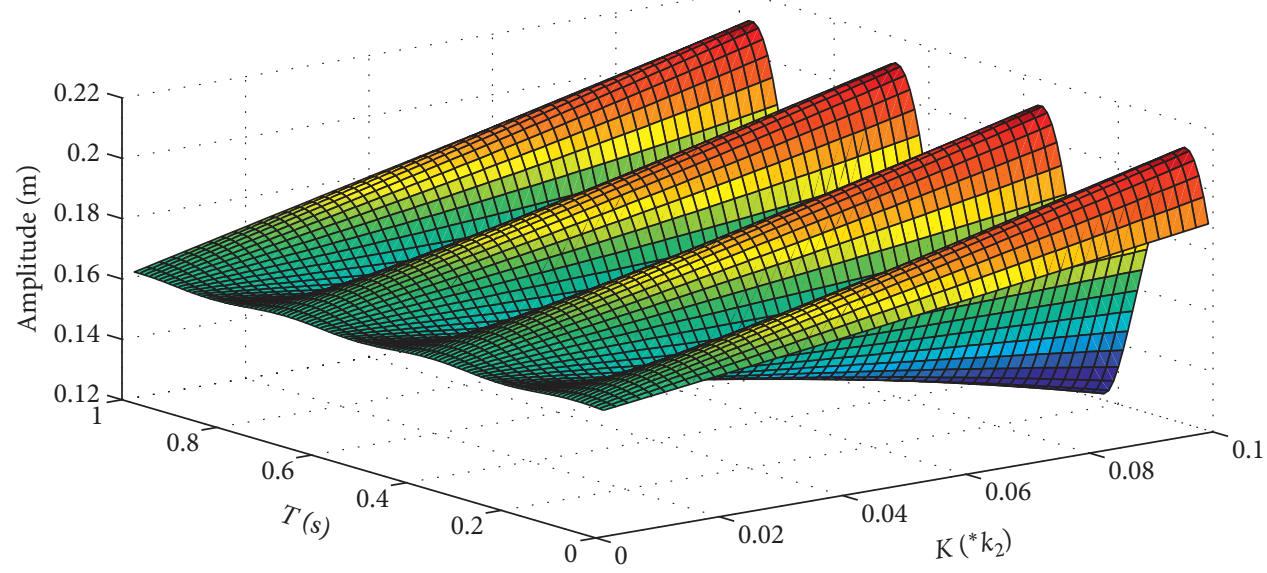

FIGURE 10: Variation of vibration amplitude of $m_{2}$ with time-delay parameter $T$ and strength parameter $K$. 


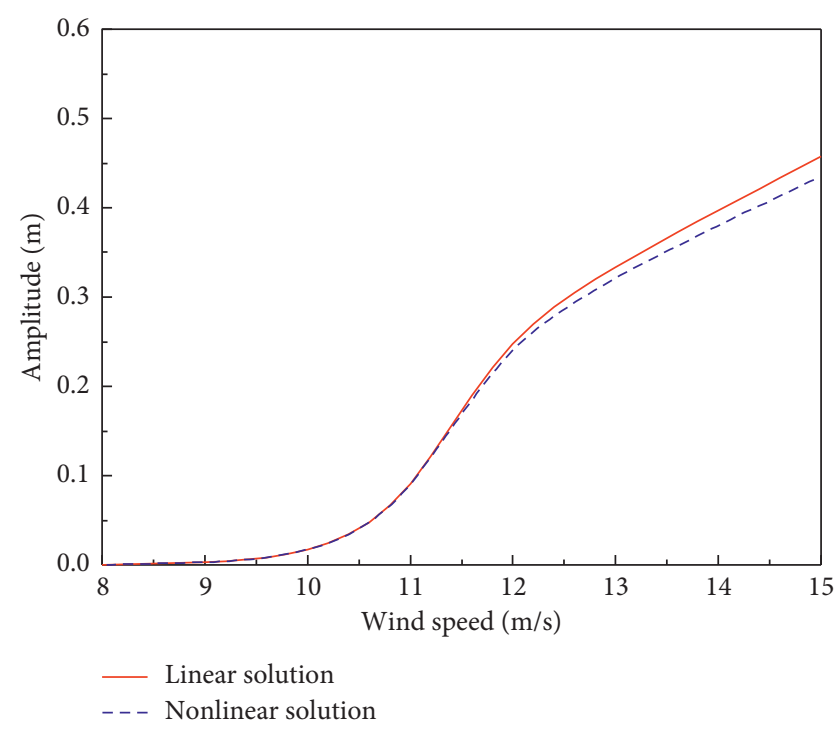

FIGURE 11: Comparison of the galloping amplitude of $m_{1}$ for linear solution and nonlinear solution.

the aerodynamic coefficients are $a_{1}=0.667, a_{2}=16.218$, and $a_{3}=-33.432$, and the air density is $\rho=1.2929 \mathrm{~kg} \cdot \mathrm{m}^{-3}$. And for first modal truncation, the equivalent mass $m_{1}$ is $149.88 \mathrm{~kg}$, the equivalent linear stiffness of iced conductors is $k_{1}=1797.44 \mathrm{~N} / \mathrm{m}$, the equivalent square nonlinear stiffness of iced conductors is $k_{12}=586.64 \mathrm{~N} / \mathrm{m}^{2}$, the equivalent cubic nonlinear stiffness of iced conductors is $k_{13}=122.85 \mathrm{~N} / \mathrm{m}^{3}$, the equivalent damping of iced conductors is $c_{1}=0.535 \mathrm{~N} \mathrm{~s} / \mathrm{m}$, the mass of the added mass is $m_{2}=0.2 m_{1}$, the stiffness of the linear spring is $k_{2}=0.1 k_{1}$, the damping coefficient of the viscous damper is $c_{2}=0.5 c_{1}$, the strength parameter of timedelayed feedback is $K=0.16 k_{2}$, and the time-delay parameter is $T=1.52 \mathrm{~s}$. The nonlinear solution is then obtained by the integration algorithm method. The linear solutions are obtained by the proposed method. It can be seen from Figure 11 that the linear solution are not too far from nonlinear solution, and the accuracy of the linear solution is enough.

\section{Conclusion}

In this paper, the effect of TTMD on the galloping control is investigated, based on the theoretical model. Three models have been introduced to contrast and solved by the harmonic balance method. Regarding the effects of the timedelay parameter $T$ and strength parameter $K$ on TTMD's vibration control, the main findings can be summarized as follows:

(1) The added fixed time-delay feedback control can promote the TTMD's effect, when suitable timedelay parameter $T$ and strength parameter $K$ are chosen.

(2) The response amplitude and natural frequency of iced conductors with TTMD system change periodically with time-delay parameter $T$.

(3) With strength parameter $K$ increasing, the response amplitude increases or decreases for a fixed value of T. However, the TTMD's effect can always be enhanced.

In future studies, parameters study should be carried out to determine the best values of $T$ and $K$.

\section{Data Availability}

The data used to support the findings of this study are available from the corresponding author upon request.

\section{Disclosure}

This paper was presented at the Proceedings of the Second International Conference on Mechanics, Materials and Structural Engineering (Beijing, China, April 14-16, 2017).

\section{Conflicts of Interest}

The authors declare that there are no conflicts of interest regarding the publication of this paper.

\section{References}

[1] A. J. Carreira, "Controlling conductor motion with interphase spacers in regions of contamination," IEEE Electrical Insulation Magazine, vol. 24, no. 6, pp. 35-42, 2008.

[2] M. L. Lu, N. Popplewell, A. H. Shah, and J. K. Chan, "Hybrid nutation damper for controlling galloping power lines," IEEE Transactions on Power Delivery, vol. 22, no. 1, pp. 450-456, 2007.

[3] M. D. Rowbottom and J. G. Allnutt, "Mechanical dampers for the contiol of full span galloping oscillations," IEE Proceedings C Generation, Transmission and Distribution, vol. 129, no. 3, pp. 123-135, 1982.

[4] Y. Guo, G. Li, and C. You, Galloping of Transmission Line, China Electric Power Press, Beijing, China, 2002.

[5] V. Gattulli and R. Ghanem, "Adaptive control of flow-induced oscillations including vortex effects," International Journal of Non-Linear Mechanics, vol. 34, no. 5, pp. 853-868, 1999.

[6] J. L. Lilien and A. A. Vinogradov, "Full-scale tests of torsional damper and detuner (TDD) antigalloping device," IEEE Transactions on Power Delivery, vol. 17, no. 2, pp. 638-643, 2002.

[7] A. Larsen, "Vortex-induced response of bridges and control by tuned mass dampers," in Structural Dynamics-EURODYN'93, T. Moan, P. G. Bergan, O. T. Gudmestad et al., Eds., pp. 1003-1010, A. A. Balkema, Rotterdam, Netherlands, 1993.

[8] A. Larsen, E. Svensson, and H. Andersen, "Design aspects of tuned mass dampers for the Great Belt East Bridge approach span," Journal of Wind Engineering and Industrial Aerodynamics, vol. 54-55, no. 2, pp. 413-426, 1995.

[9] M. D. Rowbottom, "The optimization of mechanical dampers to control self-excited galloping oscillations," Journal of Sound and Vibration, vol. 75, no. 4, pp. 559-576, 1981.

[10] Y. Fujino and M. Abé, "Design formulas for tuned mass dampers based on a perturbation technique," Earthquake Engineering and Structural Dynamics, vol. 22, no. 10, pp. 833-854, 1993.

[11] Y. Fujino, P. Warnitchai, and M. Ito, "Suppression of galloping of bridge tower using tuned mass damper," Journal of the Faculty of Engineering, vol. 38, no. 2, pp. 49-73, 1985.

[12] L. Ai-qun, Structure Vibration Control, China Machine Press, Beijing, China, 2007. 
[13] Z. N. Masoud and A. H. Nayfeh, "Sway reduction on container cranes using delayed feedback controller," Nonlinear Dynamics, vol. 34, no. 3-4, pp. 347-358, 2003.

[14] Z. N. Masoud, A. H. Nayfeh, and N. A. Nayfeh, "Sway reduction on quay-side container cranes using delayed feedback controller: simulations and experiments," Modal Analysis, vol. 11, no. 8, pp. 1103-1122, 2005.

[15] A. Mehmood, A. Abdelkefi, I. Akhtar, A. Nayfeh, A. Nuhait, and M. R. Hajj, "Linear and nonlinear active feedback controls for vortex-induced vibrations of circular cylinders," Journal of Vibration and Control, vol. 20, no. 8, pp. 1137-1147, 2012.

[16] L. Wang, W. B. Liu, and H. L. Dai, "Aeroelastic galloping response of square prisms: the role of time-delayed feedbacks," International Journal of Engineering Science, vol. 75, pp. 79-84, 2014.

[17] H. L. Dai, A. Abdelkefi, L. Wang, and W. B. Liu, "Control of cross-flow-induced vibrations of square cylinders using linear and nonlinear delayed feedbacks," Nonlinear Dynamics, vol. 78, no. 2, pp. 907-919, 2014.

[18] V. Ravichandran, V. Chinnathambi, and S. Rajasekar, "Nonlinear resonance in Duffing oscillator with fixed and integrative time-delayed feedbacks," Pramana, vol. 78, no. 3, pp. 347-360, 2012.

[19] Y. Y. Zhao and J. Xu, "Effects of delayed feedback control on nonlinear vibration absorber system," Journal of Sound and Vibration, vol. 308, no. 1-2, pp. 212-230, 2007.

[20] G. Li, L. Li, and P. Zhu, Galloping Control for Iced Conductors using Tuned Mass Dampers with Time-Delayed Feedbacks, Atlantis Press, Beijing, China, 2017, https:// download.atlantis-press.com/proceedings/icmmse-17/ 25881582.

[21] A. Barrero-Gil, G. Alonso, and A. Sanz-Andres, "Energy harvesting from transverse galloping," Journal of Sound and Vibration, vol. 329, no. 14, pp. 2873-2883, 2010.

[22] R. D. Blevins, Flow-Induced Vibrations, Van Nostrand Reinhold, New York, NY, USA, 1990.

[23] A. Luongo, D. Zulli, and G. Piccardo, "On the effect of twist angle on nonlinear galloping of suspended cables," Computers and Structures, vol. 87, no. 15-16, pp. 1003-1014, 2009.

[24] D. Vicente-Ludlam, A. Barrero-Gil, and A. Velazquez, "Enhanced mechanical energy extraction from transverse galloping using a dual mass system," Journal of Sound and Vibration, vol. 339, pp. 290-303, 2015.

[25] Electric Power Research Institute, Transmission Line Reference Book: Wind-Induced Conductor Motion, EPRI, Palo Alto, CA, USA, 1979.

[26] Q. Zhang, N. Popplewell, and A. H. Shah, "Galloping of bundle conductor," Journal of Sound and Vibration, vol. 234, no. 1, pp. 115-134, 2000. 


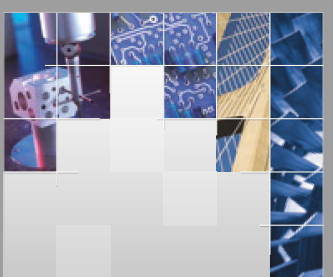

\section{Enfincering}
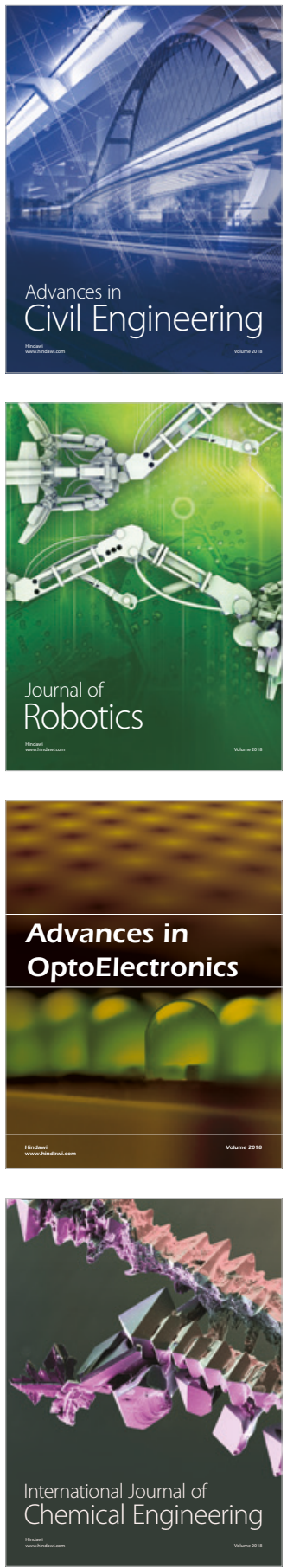

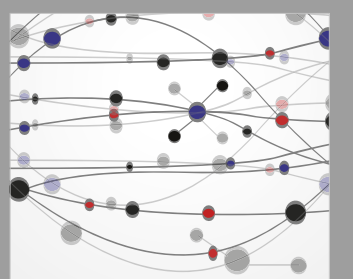

\section{Rotating \\ Machinery}

The Scientific World Journal

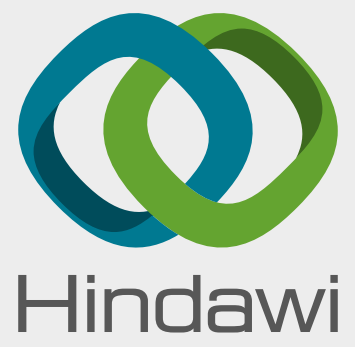

Submit your manuscripts at

www.hindawi.com
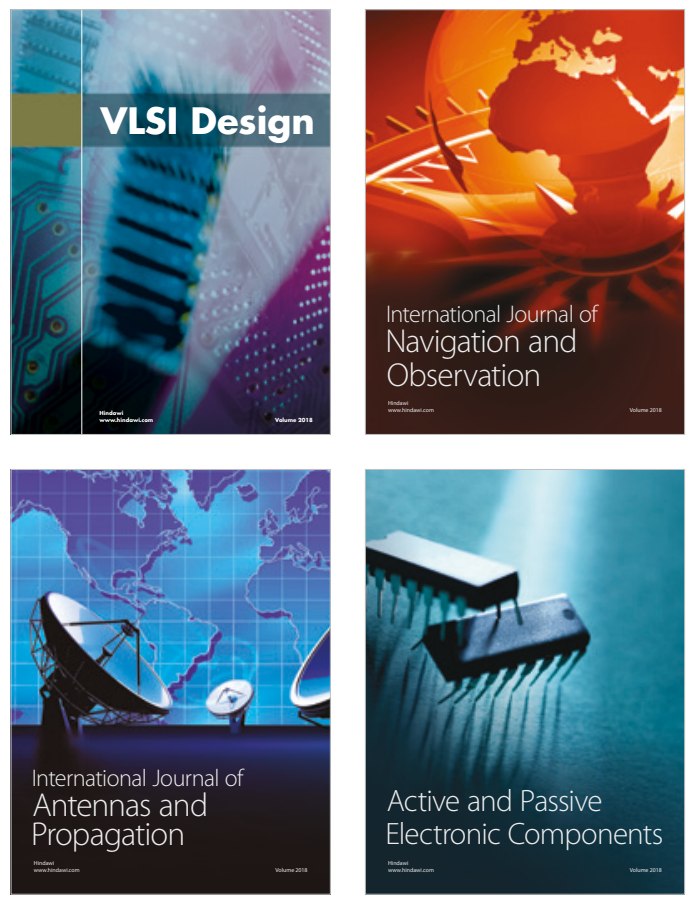
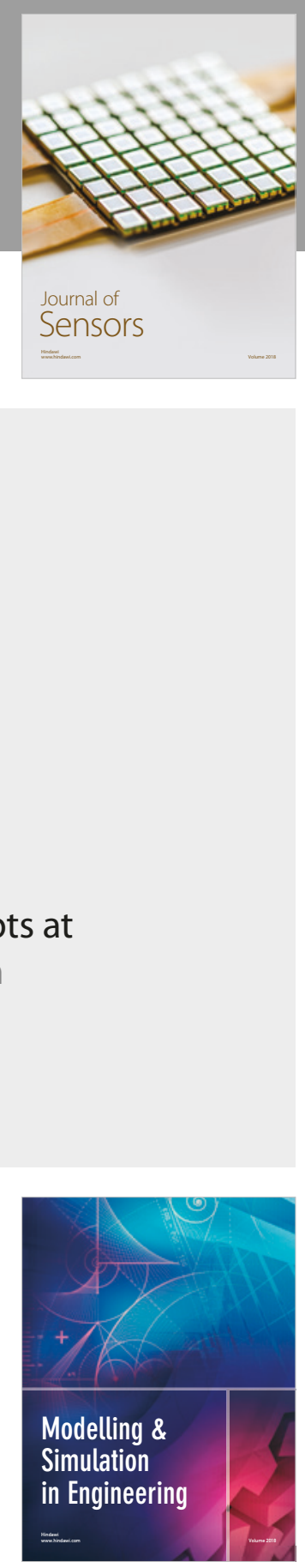

\section{Advances \\ Multimedia}
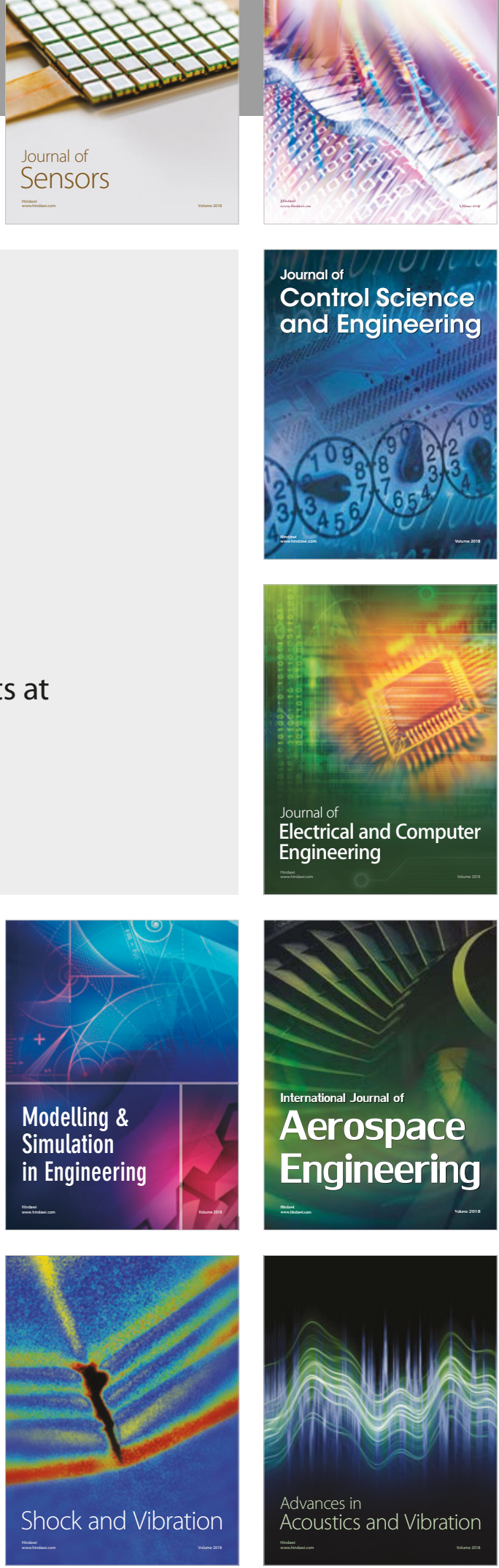